\title{
Analysis of tissue surrounding thyroid nodules by ultrasound digital images
}

\author{
Pierpaolo Trimboli • Fabiano Bini - Massimiliano Andrioli • Luca Giovanella • \\ Maria Francesca Thorel - Luca Ceriani - Stefano Valabrega • Andrea Lenzi • \\ Francesco Maria Drudi · Franco Marinozzi · Francesco Romanelli
}

Received: 3 April 2014/ Accepted: 18 June 2014

(C) Springer Science+Business Media New York 2014

\begin{abstract}
Since US is not easily reproducible, the digital image analysis (IA) has been proposed so that the image evaluation is not subjective. In fact, IA meets the criteria of objectivity, accurateness, and reproducibility by a matrix of pixels whose value is displayed in a gray level. This study aims at evaluating via IA the tissue surrounding a thyroid nodule (backyard tissue, BT) from goitres with benign (bBT) and malignant (m-BT) lesions. Sixty-nine US images of thyroid nodules surrounded by adequate thyroid tissue was classified as normoechoic and homogeneous were enrolled as study group. Forty-three US images from normal thyroid (NT) glands were included as controls. Digital images of $800 \times 652$ pixels were acquired at a resolution of eight bits with a 256 gray levels depth. By one-way ANOVA, the 43 NT glands were not statistically different $(P=0.91)$. Mean gray level of normal glands was
\end{abstract}

P. Trimboli and F. Bini contributed equally to this work.

P. Trimboli $(\square)$

Section of Endocrinology and Diabetology, Ospedale Israelitico

of Rome, Via Fulda, 14, 00148 Rome, Italy

e-mail: pierpaolo.trimboli@gmail.com

F. Bini · F. Marinozzi

Department of Aerospace and Mechanical Engineering,

Sapienza University of Rome, Rome, Italy

M. Andrioli

EndocrinologiaOggi, Rome, Italy

L. Giovanella $\cdot$ L. Ceriani

Department of Nuclear Medicine and Thyroid Centre, Oncology

Institute of Southern Switzerland, Bellinzona, Switzerland

M. F. Thorel

Section of Ultrasonography, Ospedale Israelitico of Rome,

Rome, Italy significantly higher than $\mathrm{b}-\mathrm{BT}(P=0.026)$, and $\mathrm{m}$-BT $(P=0.0001)$, while no difference was found between b-BT and m-BT $(P=0.321)$. NT tissue boundary external to the nodule was found at $6.0 \pm 0.5 \mathrm{~mm}$ in cancers and $4.0 \pm 0.5 \mathrm{~mm}$ in benignancies $(P=0.001)$. These data should indicate that the tissue surrounding a thyroid nodule may be damaged even when assessed as normal by US. This is of interest to investigate the extranodular effects of thyroid tumors.

Keywords Thyroid · Ultrasonography · Digital image analysis

\section{Introduction}

Ultrasonography (US) is recognized as the main tool in assessing thyroid gland morphology and size $[1,2]$. The

\author{
S. Valabrega \\ Department of Surgical and Medical Sciences, Ospedale S. \\ Andrea, Sapienza University of Rome, Rome, Italy \\ A. Lenzi · F. Romanelli \\ Department of Experimental Medicine, Sapienza University of \\ Rome, Rome, Italy \\ F. M. Drudi \\ Department of Radiological Sciences, Sapienza University of \\ Rome, Rome, Italy
}


majority of papers on thyroid US focused on nodule's risk features and selection for biopsy [3-6]. Few studies analyzed the relation between thyroid echographic appearance and its function. These papers report that hypoechogenicity of the thyroid gland represents a risk factor for developing hypothyroidism over time [7-10]. Also, at least $80 \%$ of subjects with normal thyroid (NT) US pattern have NT function [11, 12]. To recap, US examination is characterized by poor reproducibility due to high intra- and inter-observer variability $[13,14]$. As a consequence, the potential standardization of US exam still remains a challenge.

The digital image analysis (IA) has been proposed to overcome the observer variability of image evaluation. In fact, IA meets the criteria of objectivity, accurateness, and reproducibility $[15,16]$. The digitalized US image is composed of a matrix of numbers where each element corresponds to a "picture element" (pixel) and whose value is displayed in a gray level. This gives the echogenicity of the tissue in that point.

Here, we aimed to evaluate by IA the backyard tissue (BT) of thyroid lesions, such as not nodular thyroid tissue surrounding benign and malignant nodules. The study included a large series of nodular US images, and thyroid disease-free subjects as a control group.

\section{Materials and methods}

\section{Selection of the series}

The study group initially comprised 95 cases from 95 patients undergone histologic and/or cytologic examination. NT function and negative thyroid autoimmunity were proved in all subjects. All US images had a thyroid nodule surrounded by extranodular tissue that was classified as homogeneous and normoechoic by two observers (see below). A series of 13 cases were excluded because of small tissue area around the nodule, seven cases were excluded because the images were not clear for the analysis, and other six cases were disagreeing between the US examiners. Then, the final series included 23 malignant nodules and 46 benign ones surrounded by adequate not nodular tissue. Cancer group included 22 papillary cancers (9 classic, 11 follicular variant, 1 tall cell, 1 sclerosing variant) and one minimally invasive follicular cancer. As controls, 43 images of NT gland from euthyroid subjects were enrolled (see below).

Thyroid ultrasound evaluation

Two expert examiners (PT, MFT) performed US using an Esaote MyLab system (Esaote, Genova, Italy) equipped with linear probe working at $8-14 \mathrm{MHz}$. The inter- observer variability for these investigators was $8.2 \%[11$, 12]. To evaluate gland echogenicity and homogeneity, clips and freeze images for all patients were stored and blindly reviewed by the two investigators. The discordant cases were not included in the study.

Analysis of thyroid ultrasound images

Digital images of $800 \times 652$ pixels were acquired at a resolution of eight bits with a 256 gray levels depth. The IA was initially tested in 43 thyroid US images carried out from 43 subjects. The latter had been assessed as thyroid disease free because of normal serum thyroid hormones and negative anti-thyroid antibodies combined with not nodular thyroid gland. This series was included in the study as control group (NT). System parameters (transducer properties, near and far gain, depth gain compensation, pulse intensity and storage method) were kept constant. Consequently, it should be assumed that changes in echographic texture are directly related to changes in pathology.

To analyze the BT, an annular region of interest (ROI) around the nodule was selected. The outer boundary of each nodule which delimits the first circular ROI, referred to as forward tissue, was delineated by following the rule that a pixel belongs to the nodule when it is included in at least two out of the three delineations drawn by the physician. The second annular ROI is the BT of each nodule which was selected by following the rule of normalizing the BT area to the ROI including the nodule. In particular, the BT area was chosen 3.5 times greater than to the nodule ROI, i.e., $A \mathrm{Ara}_{\mathrm{BT}} / \mathrm{Area}_{\text {forward tissue }}=3.5$.

Therefore, we employed a set of reference-standard algorithms and graphical tools for image processing, analysis, and visualization. The software development environment was VISION ${ }^{\circledR}$ toolbox of the LabView ${ }^{\circledR}$ platform (LabView 2013, National Instruments, Austin, TX, USA) which was used for the analysis of the digital images.

To quantify the textural changes, the first-order statistics, such as the mean and the standard deviation of the gray levels were computed from the histogram $H(j)$ of the two ROIs for each image. The cumulative density function is defined as

$H(j)=\sum_{i=0}^{j} h(i)$

$(j=0,1, \ldots, 255)$ where the histogram $h(i)$ with $i=0, \ldots$, 255 is:

$h(i)=\frac{\text { number of pixels of gray level }(i)}{\text { total number of pixels }}$

To evaluate further the potential extension of the BT, we also analyzed other ROIs, with the shape of random quarter 
circle segment with a radius of $2.0 \pm 0.5 \mathrm{~mm}$ external to the BT, indicated as the bridge area. The latter first boundary, in which the gray levels were not different from those recorded in the controls, was established as the first normal tissue surrounding the nodule.

\section{Statistical analysis}

Statistical analysis was performed using SSPS v 20.0 software (Chicago, IL, USA). The studied groups were the followings: benign nodules, malignant nodules, BT of the benign nodules (b-BT), BT of the malignant nodules (m$\mathrm{BT}$ ), and normal tissue (NT).

The normality for continuous variables in the group was determined by the Shapiro-Wilk Test. The variables showed a normal distribution $(P>0.05)$. A one-way analysis of variance (ANOVA) was used for the comparison of variables among the studied groups.

A Bonferroni test was used to assess the differences in the Gray levels values for the two-tailed test. A value of $P<0.05$ was considered significant.

The studied groups results were given as pooled mean \pm pooled standard deviation and pooled standard error computed with $95 \%$ confidence level.

\section{Results}

The one-way ANOVA was used for the comparison of variables among the 43 NT glands and there was not statistical difference $(P=0.91)$.

Mean gray levels of b-BT, m-BT, and NT groups were significantly different $(P=0.0001)$. Post-hoc tests revealed significant differences between $\mathrm{b}-\mathrm{BT}$ and NT $(P=0.026)$, and $\mathrm{m}-\mathrm{BT}$ and NT $(P=0.0001)$, while there were no significant difference between $\mathrm{b}-\mathrm{BT}$ and $\mathrm{m}-\mathrm{BT}$ $(P=0.321)$ (Fig. 1).

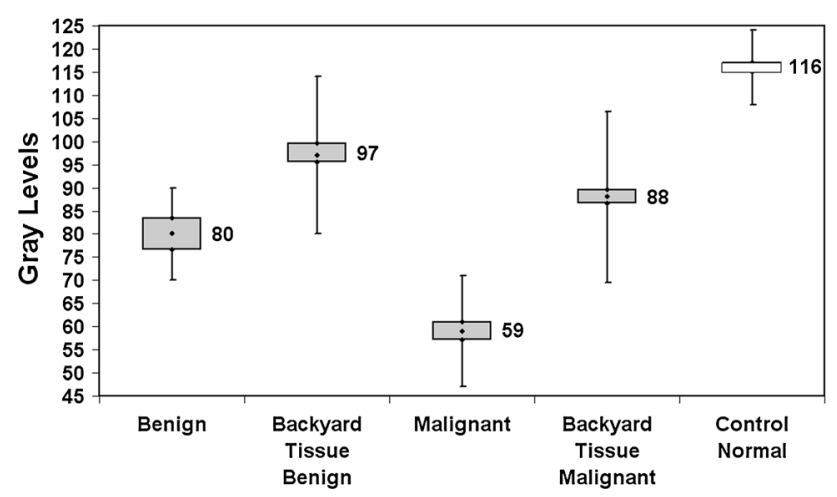

Fig. 1 Pooled mean, standard deviation and standard error of gray levels values recorded in the study groups and controls
The gray levels value of benign nodules, cancers, and NT was significantly different $(P=0.0001)$. Post-hoc tests revealed significant differences between benign lesions and NT $(P=0.002)$, and cancers and NT $(P=0.0001)$. No significant difference between benignancies and malignancies $(P=0.529)$ was recorded.

As for the first NT tissue boundary external to the BT, a significant $(P=0.001)$ difference was found between the bridge area of the malignant and benign nodules. Specifically, cancers showed a bridge area tissue with gray levels not different with respect to the controls at $6.0 \pm 0.5 \mathrm{~mm}$, while benign lesions at $4.0 \pm 0.5 \mathrm{~mm}$. No significant correlation was found between the size of damaged BT and nodule's volume or cancer histologic type.

Finally, post-hoc tests showed significant difference between benign nodules and b-BT $(P=0.001)$, and between cancers and $\mathrm{m}-\mathrm{BT}(P=0.017)$.

\section{Discussion}

US allows to evaluate thyroid structure and volume, as well as to discover non-palpable lesions which should be undetectable using other techniques $[1,2,17,18]$. Nonetheless, echographic examination is strongly influenced by observer variability and it is affected by poor reproducibility [19]. The analysis of digital images has been reported as accurate $[15,16]$. In particular, imaging analysis is used for the extraction of clinical data and it is used to processing digital images by means of computer algorithms.

Here, we aimed at investigating the not nodular thyroid tissues from malignant and benign nodules via IA. Our main result is the following one. While all 112 not nodular tissue images (69 extranodular samples and 43 NTs) were assessed as normoechoic and homogeneous by two experienced US examiners, the surrounding tissues of benign and malignant nodules had lower gray levels with respect to those obtained in controls. Interestingly, the tissues surrounding benign and malignant nodules were not different at real time US as well as at IA. Regarding the latter finding, we can speculate that the damage surrounding thyroid nodules represents the result of mechanical changes involving the stroma adjacent to thyroid lesions. Adjunctive studies on this field, possibly supported by elastographic evaluation [20], are needed to confirm this hypothesis.

However, the present data should indicate that cancers have a more extended damage than benign lesions ( 6 and $4 \mathrm{~mm}$, respectively). The latter finding should achieve a high significance in clinical practice. Based on these data, we can speculate that a thyroid cancer near the margins of the gland should have a more chance of invasion of gland capsule and extension out of that. Whether thyroid tumor 
affects the surrounding thyroid tissue is unknown, and very sparse histologic data have been reported. In particular, Wang et al. [21] described a computerized analysis of digital images to detect thyroid follicular adenoma or carcinoma from normal gland, with interesting results. The results recorded from US images are in agreement with those of histologic findings, as well as with advice for further ultrasonographic studies. Some studies showed that in multinodular goitres clonal and polyclonal nodules coexist. Interestingly, nodules in recurrent goitres are predominantly polyclonal, while many thyroid cancers are polyclonal [22-24]. The collected data suggest that both benign and malignant nodules have their origin in a de novo proliferation of different cohorts of thyrocytes supporting the presence of subtle alterations within the clinically and sonographically normal perinodular tissue.

Some limits of the paper have to be briefly discussed. Nodules included in the study have been undergone fineneedle aspiration cytology (and surgery, in a part of these). Thus, nodule's hypoechogenicity, known as a main risk factor for thyroid malignancy, might be more prevalent in the series, and nodule's echogenicity was no significantly different between cancer and benign group. In addition, future studies are needed to verify the herein presented IA in thyroid nodules with different US presentations (i.e. micro- and macrocalcifications, cystic changes).

In conclusion, this study suggests that the tissue surrounding a thyroid lesion may be damaged even when it is assessed as normal by real time ultrasound examination. Also, the damaged annulus is larger in malignant than in benign nodules. This is of interest to investigate the potential extranodular effects of thyroid tumors.

Conflict of interest The authors declare no conflict of interest.

\section{References}

1. W. Blank, B. Braun, Sonography of the thyroid-part 1. U1traschall Med. 28, 554-568 (2007)

2. W. Blank, B. Braun, Sonography of the thyroid—part 2: thyroid inflammation, impairment of thyroid function and interventions. Ultraschall Med. 29, 128-149 (2008)

3. E. Papini, R. Guglielmi, A. Bianchini, A. Crescenzi, S. Taccogna, F. Nardi, C. Panunzi, R. Rinaldi, V. Toscano, C.M. Pacella, Risk of malignancy in nonpalpable thyroid nodules: predictive value of ultrasound and color-Doppler features. J. Clin. Endocrinol. Metab. 87, 1941-1946 (2002)

4. T. Rago, P. Vitti, L. Chiovato, S. Mazzeo, A. De Liperi, P. Miccoli, P. Viacava, F. Bogazzi, E. Martino, A. Pinchera, Role of conventional ultrasonography and color flow-doppler sonography in predicting malignancy in 'cold' thyroid nodules. Eur. J. Endocrinol. 138, 41-46 (1998)

5. P. Trimboli, R. Guglielmi, S. Monti, I. Misischi, F. Graziano, N. Nasrollah, S. Amendola, S.N. Morgante, M.G. Deiana, S.
Valabrega, V. Toscano, E. Papini, Ultrasound sensitivity for thyroid malignancy is increased by real-time elastography: a prospective multicenter study. J. Clin. Endocrinol. Metab. 97, 4524-4530 (2012)

6. H. Gharib, E. Papini, R. Paschke, D.S. Duick, R. Valcavi, L. Hegedüs, P. Vitti, AACE/AME/ETA Task Force on Thyroid Nodules, American Association of Clinical Endocrinologists, Associazione Medici Endocrinologi, and European Thyroid Association Medical Guidelines for clinical practice for the diagnosis and management of thyroid nodules. Endocr. Pract. 16(Suppl. 1), 1-43 (2010)

7. T. Rago, L. Chiovato, L. Grasso, A. Pinchera, P. Vitti, Thyroid ultrasonography as a tool for detecting thyroid autoimmune diseases and predicting thyroid dysfunction in apparently healthy subjects. J. Endocrinol. Invest. 24, 763-769 (2001)

8. C. Marcocci, P. Vitti, F. Cetani, F. Catalano, R. Concetti, A. Pinchera, Thyroid ultrasonography helps to identify patients with diffuse lymphocytic thyroiditis who are prone to develop hypothyroidism. J. Clin. Endocrinol. Metab. 72, 209-213 (1991)

9. T. Rago, G. Di Coscio, C. Ugolini, M. Scutari, F. Basolo, F. Latrofa, R. Romani, P. Berti, L. Grasso, L.E. Braverman, A. Pinchera, P. Vitti, Clinical features of thyroid autoimmunity are associated with thyroiditis on histology and are not predictive of malignancy in 570 patients with indeterminate nodules on cytology who had a thyroidectomy. Clin. Endocrinol. (Oxf.) 67, 363-369 (2007)

10. P.W. Rosário, B. Bessa, M.M. Valadão, S. Purisch, Natural history of mild subclinical hypothyroidism: prognostic value of ultrasound. Thyroid 19, 9-12 (2009)

11. P. Trimboli, F. Rossi, E. Condorelli, O. Laurenti, C. Ventura, G. Nigri, F. Romanelli, M. Guarino, S. Valabrega, Does normal thyroid gland by ultrasonography match with normal serum thyroid hormones and negative thyroid antibodies? Exp. Clin. Endocrinol. Diabetes 118, 630-632 (2010)

12. P. Trimboli, F. Rossi, F. Thorel, E. Condorelli, O. Laurenti, C. Ventura, G. Nigri, F. Romanelli, M. Guarino, S. Valabrega, One in five subjects with normal thyroid ultrasonography has altered thyroid tests. Endocr. J. 59, 137-143 (2012)

13. W. Shabana, E. Peeters, P. Verbeek, M.M. Osteaux, Reducing inter-observer variation in thyroid volume calculation using a new formula and technique. Eur. J. Ultrasound. 16, 207-210 (2003)

14. M.B. Zimmermann, L. Molinari, M. Spehl, J. Weidinger-Toth, J. Podoba, S. Hess, F. Delange, Toward a consensus on reference values for thyroid volume in iodine-replete schoolchildren: results of a workshop on inter-observer and inter-equipment variation in sonographic measurement of thyroid volume. Eur. J. Endocrinol. 144, 213-220 (2001)

15. C. Skouroliakou, M. Lyra, A. Antoniou, L. Vlahos, Quantitative image analysis in sonograms of the thyroid gland. Nucl. Instrum. Methods Phys. Res. 569, 606-609 (2006)

16. F. Marinozzi, F.P. Branca, F. Bini, A. Scorza, Calibration procedure for performance evaluation of clinical pulsed Doppler systems. Measurement 45, 1334-1342 (2012)

17. P. Vitti, T. Rago, Thyroid ultrasound as a predicator of thyroid disease. J. Endocrinol. Invest. 26, 686-689 (2003)

18. P. Trimboli, M. Ruggieri, A. Fumarola, M. D’Alò, A. Straniero, A. Maiuolo, S. Ulisse, M. D'Armiento, A mathematical formula to estimate in vivo thyroid volume from two-dimensional ultrasonography. Thyroid 18, 879-882 (2008)

19. S.J. Park, S.H. Park, Y.J. Choi, D.W. Kim, E.J. Son, H.S. Lee, J.H. Yoon, E.K. Kim, H.J. Moon, J.Y. Kwak, Interobserver variability and diagnostic performance in US assessment of thyroid nodule according to size. Ultraschall Med. 33, 186-190 (2012)

20. M. Andrioli, L. Persani, Elastographic techniques of thyroid gland: current status. Endocrine (2014). doi:10.1007/s12020-0140178-1 
21. W. Wang, J.A. Ozolek, G.K. Rohde, Detection and classification of thyroid follicular lesions based on nuclear structure from histopathology images. Cytometry 77, 485-494 (2010)

22. P. Harrer, M. Bröcker, A. Zint, M. Derwahl, L. Barbera, V. Zumtobel, The clonality of nodules in recurrent goiters at second surgery. Langenbecks Arch. Surg. 383, 453-455 (1998)
23. T.M. Shattuck, W.H. Westra, P.W. Ladenson, A. Arnold, Independent clonal origins of distinct tumor foci in multifocal papillary thyroid carcinoma. N. Engl. J. Med. 352, 2406-2412 (2005)

24. F.A. Verburg, M. Dietlein, M. Lassmann, M. Luster, C. Reiners, Why radioiodine remnant ablation is right for most patients with differentiated thyroid carcinoma. Eur. J. Nucl. Med. Mol. Imaging 36, 343-346 (2009) 